\title{
An efficient quantum search engine on unsorted database
}

\author{
Heping $\mathrm{Hu}$ * Y Yingyu Zhang, and Zhengding Lu \\ Huazhong University of Science and Technology
}

(Dated: September 12, 2018)

\begin{abstract}
We consider the problem of finding one or more desired items out of an unsorted database. Patel has shown that if the database permits quantum queries, then mere digitization is sufficient for efficient search for one desired item. The algorithm, called factorized quantum search algorithm, presented by him can locate the desired item in an unsorted database using $O\left(\log _{4} N\right)$ queries to factorized oracles. But the algorithm requires that all the property values must be distinct from each other. In this paper, we discuss how to make a database satisfy the requirements, and present a quantum search engine based on the algorithm. Our goal is achieved by introducing auxiliary files for the property values that are not distinct, and converting every complex query request into a sequence of calls to factorized quantum search algorithm. The query complexity of our algorithm is $O\left(P * Q * M * \log _{4} N\right)$, where $\mathrm{P}$ is the number of the potential simple query requests in the complex query request, $\mathrm{Q}$ is the maximum number of calls to the factorized quantum search algorithm of the simple queries, $\mathrm{M}$ is the number of the auxiliary files for the property on which our algorithm are searching for desired items. This implies that to manage an unsorted database on an actual quantum computer is possible and efficient.
\end{abstract}

\section{INTRODUCTION}

Consider a collection of items in a database, characterized by some property with values on the real line. Sorting these items means arranging them in an ordered sequence according to their property values. The purpose of sorting is to facilitate subsequent searches of that database for items with known values of the property [1].

The best classical algorithm uses $\log _{2} N$ queries to find a desired item out of an ordered list of $\mathrm{N}$ items, while a quantum computer can solve the problem using a constant factor fewer queries. The best known lower bound shows that any quantum algorithm for the problem requires at least $(\ln N-1) / \pi \approx 0.221 \log _{2} N$ queries [2]. The algorithm obtained by Farhi, Goldstone, Gutmann, and Sipser, uses $3\left\lceil\log _{52} N\right\rceil$ queries, showing that a constant factor speedup is indeed possible [3]. The best published exact quantum algorithm for the problem uses $4 \log _{605} N \approx 0.433 \log _{2} N$ queries [4].

In this paper, we consider the problem of finding one or more desired items out an unsorted database. Patel has shown that if the database quantum queries, then mere digitization is sufficient for efficient search for one desired item. The algorithm, called factorized quantum search algorithm, presented by him can locate the desired item in an unsorted database using $O\left(\log _{4} N\right)$ queries to facorized oracles [1]. Unfortunately, the algorithm would not work if it is used to search more than one desired items out of an unsorted database, or the property values among which it is searching for the desired item are not distinct. From the point of view of unsorted database manipulation, we consider the problem with more than one desired items in an unsorted database and with the property values that are not distinct from each other.

*e-mail: newpine2002@yahoo.com.cn
And we present a quantum search engine, based on the factorized quantum search algorithm, that can solve the problems. Our goal is achieved by introducing auxiliary files for the property values that are not distinct, and converting the searching request into a sequence of calls to factorized quantum search algorithm.

The paper is organized as follows: In section II, we review quantum random access memory, Structured Query Language, and factorized quantum search algorithm. In section III, we present our quantum search engine, discuss how to digitize the property values of an unsorted database, which might be distinct from each other or not, and discuss how to convert a complex query request into a sequence of calls to the factorized quantum search algorithm. We also discuss how the factorized quantum search algorithm works on an actual quantum computer with the unsorted database existing in the quantum random access memory. In section IV, We give several examples to show how the quantum search engine works. We conclude the paper in section $\mathrm{V}$.

\section{BACKGROUNDS}

\section{A. Quantum random access memory}

A quantum random access memory (qRAM) uses nqubits to address any quantum superposition of $\mathrm{N}$ memory cells. It means the qRAM can perform memory accesses in coherent quantum superposition [5]: if the quantum computer needs to access a superposition of memory cells, the address register A must contain a superposition of addresses $\sum_{j} a_{j}|j\rangle_{A}$, and the qRAM will return a superposition of data in a data register $\mathrm{D}$, correlated with the address register:

$$
\sum_{j} a_{j}|j\rangle_{A} \longrightarrow \sum_{j} a_{j}|j\rangle_{A}\left|d_{j}\right\rangle_{D}
$$


where $d_{j}$ is the content of the jth memory cell []. Giovannetti, Lloyd and Maccone have presented an architecture that exponentially reduces the requirements for a memory call: $O\left(\log _{2} N\right)$ switches need be thrown instead of the $\mathrm{N}$ used in classical RAM designs, which yields a more robust qRAM algorithm than conventional (classical and quantum) designs. See [6] for more details.

\section{B. Structured query language}

Structured Query Language (SQL) is a tool widely used in manipulating the classical databases [7].Basic operations in SQL include inserting a new record to a database file (INSERT), updating an existing record (UPDATE), deleting an exiting record (DELETE), selecting (SELECT) and performing an arbitrary operation on some records, backing up a portion of a database (BACKUP). and restoring the backup (RESTORE) [8]. The most commonly used operation among them might be the SELECT operation, which is defined as:

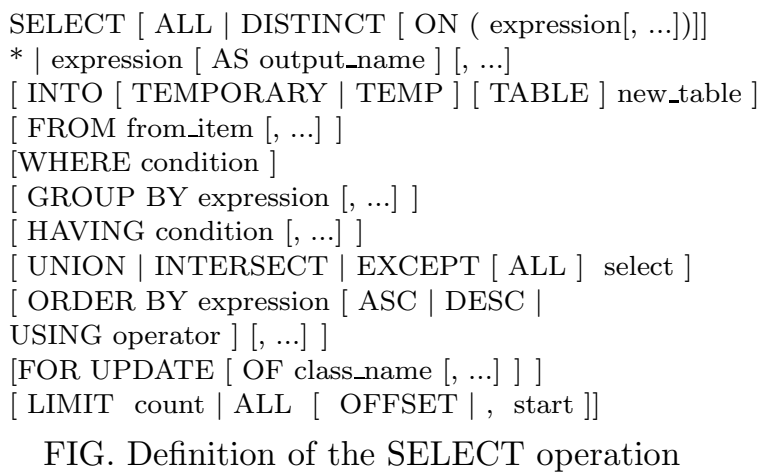

The SELECT statement is used to form queries for extracting information out of the database. The details about how to use it and the other basic operations can be found in [7]. We only show several examples, about the SELECT operation, useful for our discussion below. Assume that we have such a table as follows stored in the database, and the name of the table is Table1.

\begin{tabular}{|c|c|c|}
\hline ID & Name & Age \\
\hline 960112 & Lin & 18 \\
\hline 960114 & Yang & 20 \\
\hline 960113 & Xing & 19 \\
\hline 960115 & Yingyu & 20 \\
\hline
\end{tabular}

Table. 1

Example 1:

SELECT ID, Name, Age

FROM Table1

WHERE Age $=20$

It means selecting the records whose property values of Age is 20. This operation will return with

\begin{tabular}{|c|c|c|}
\hline ID & Name & Age \\
\hline 960114 & Yang & 20 \\
\hline 960115 & Yingyu & 20 \\
\hline
\end{tabular}

Table. 2: Result of example 1.

Example 2:

SELECT ID, Name, Age

FROM Table1

WHERE ID $<960115$

Similarly, this operation will return with

\begin{tabular}{|c|c|c|}
\hline ID & Name & Age \\
\hline 960112 & Lin & 18 \\
\hline 960114 & Yang & 20 \\
\hline 960113 & Yang & 19 \\
\hline
\end{tabular}

Table. 3: Result of example 2.

Example 3:

SELECT ID, Name, Age

FROM Table1

WHERE ID $<960115$ AND Age $=20$

It will return with

\begin{tabular}{|c|c|c|}
\hline ID & Name & Age \\
\hline 960114 & Yang & 20 \\
\hline
\end{tabular}

Table. 4: Result of example 3.

\section{Factorized quantum search algorithm}

In [1], Patel has observed that sorting can be thought of as factorization of the search process and the location of a desired item in a sorted database can be found by classical queries that inspect one letter of the label at a time. Consider a collection of items in a database, characterized by some property with values on the real line. Sorting these items means arranging them in an ordered sequence according to their property values. In order to efficiently implement the sorting algorithm we need to digitize the property values. It means replacing the property values by integer labels and writing them as a string of letters belonging to a finite alphabet. In digital computers, this finite alphabet has size 2 , and the letters are called bits [1]. Assume that the database have $N=2^{n}$ items (If $\mathrm{N}$ is not a power of 2 , then the database is padded up with extra labels to make $N=2^{n}$.) and without loss of generality the desired item has the label

$$
x \equiv x_{1} x_{2} \ldots x_{n}=00 \ldots 0 .
$$


Then the search process is equivalent to finding $\mathrm{x}$ such that

$$
f(x)=\prod_{i} f_{i}\left(x_{i}\right)=\left(1-x_{1}\right)\left(1-x_{2}\right) \ldots\left(1-x_{n}\right)
$$

equals to one. In the sorted and digitized database, one searches for an item by inspecting only one $x_{i}$ at a time, i.e., evaluating $\mathrm{f}(\mathrm{x})$ by sequentially combining its factors $f_{i}\left(x_{i}\right)$. The functions $f(x)$ and $f_{i}\left(x_{i}\right)$ are referred to as oracle and factorized oracle respectively. If only the oracle $f(x)$ is available then the search process requires $O(N / 2)$ queries even with a sorted database. The collection of factorized oracles $f_{i}\left(x_{i}\right)$ is more powerful than the global oracle $f(x)$ (One can construct $\mathrm{f}(\mathrm{x})$ by combining all the $f_{i}\left(x_{i}\right)$ together.). And with the factorized oracles, the search process requires only $O(\ln N)$ queries [1].

In the paper, Patel has also observed that if the database permits quantum queries, then mere digitization is sufficient for efficient search with the quantum factorized oracles. The algorithm called factorized quantum search algorithm introduced by him is described as

$$
|x\rangle=\prod_{i=1}^{n}\left(P_{i} R_{i} F_{i}\right)\left|\psi_{\text {start }}\right\rangle,
$$

where $\left|\psi_{\text {start }}\right\rangle=\sum_{j=1}^{n} \frac{1}{\sqrt{N}}|j\rangle$, which is the uniform superposition of all the $\mathrm{N}$ states. The transformations $F_{i}$, $R_{i}$, and $P_{i}$ act on a single letter of the label only. $F_{i}$ will evaluate to -1 when the letter and its corresponding letter of the desired item match and to +1 when they do not.

$$
F_{i}=I_{1} \otimes \ldots \otimes\{ \pm 1\}_{i} \otimes \ldots \otimes I_{n}
$$

The reflection operator $R_{i}$ can also written in a factorized form,

$$
R_{i}=I_{1} \otimes \ldots \otimes\left\{R_{0}\right\}_{i} \otimes \ldots \otimes I_{n}
$$

where

$$
R_{0}=\frac{1}{2}\left(\begin{array}{llll}
-1 & +1 & +1 & +1 \\
+1 & -1 & +1 & +1 \\
+1 & +1 & -1 & +1 \\
+1 & +1 & +1 & -1
\end{array}\right)
$$

The projection/measurement operator $P_{i}$ removes from the Hilbert space all the states with zero amplitude. Note that the quantum database is digitized with the size of the alphabet equals to 4 . The factorized quantum search algorithm locates the desired item in an unsorted database using $O\left(\log _{4} N\right)$ queries.

The search process inspects each letter of the label in turn, and decide whether it matches the corresponding letter of the desired string or not. The algorithm use Grover's algorithm [9, 10] to do this job. Since Grover's algorithm requires only one query to pick one item out of four with certainty, it is efficient to digitize the quantum database with the alphabet $\{|00\rangle,|01\rangle,|10\rangle,|11\rangle\}$. Digitization requires that the items must be distinct from each other, and before digitization, we might need to pad up the database. We will turn back to these two problems later.

\section{THE QUANTUM SEARCH ENGINE}

\section{A. The function of the engine}

The database we are going to searching for desired items is unsorted. It is the only difference between the database and a general database. Our quantum search engine on such a database can be demonstrated by FIG.1, in which the Implementation module in charge of carrying out an actual quantum database search algorithm (i.e. Factorized quantum search algorithm here). The purpose of the analysis module is to resolve the query conditions passed into the engine by outside programs such as database application programs, and initiates a sequence of calls to the implementation module.

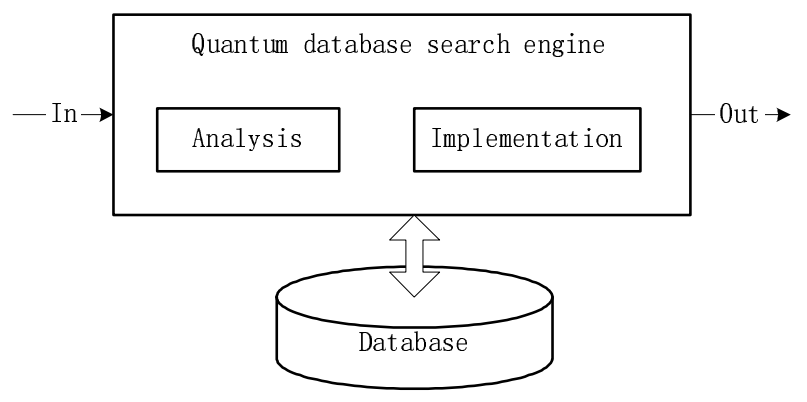

FIG. 1: The function map of quantum search engine

We will return to the implementation module later, but in the rest of this subsection, we focus on the analysis module. From the previous section we can see that mere digitalization is sufficient for the factorized quantum search algorithm. The digitalization requires that the property values are distinct from each other. It means that we can not apply the factorized quantum search algorithm directly if there are more than one potentially desired items in the query conditions. For example, the query indicated by the example 1 in section II can not carried out directly by implementing the factorized quantum search algorithm, because query condition of it includes 2 potential desired records. We have used the fact that if one implements a query on a database management interface(Here, one query means a SQL statement with a query condition, which is different from the "query" used in query complexity theory), the corresponding query request will be sent to the search engine.

The problem that how to digitize a database becomes how many potential target items in one query. To answer this problem we need to make query request clearer.

Definition 1. A complex query is a SQL statement with 
a relational expression.

Definition 2. A simple query is a SQL statement with a logical expression.

A query condition is equivalent to a relational expression, which combines two or more logical expressions with relational operators such as AND, OR and NOT. A logical expression is an expression that has two operands connected with a logical operator from the set $\{>, \geqslant,<, \leqslant,=, \neq\}[8]$.

Theorem 1. Any complex query can be split into a sequence of simple queries.

To prove this theorem we need three facts:

Fact 1: Assume that a query condition has the form of "CONDITION1 AND CONDITION2". We can divided it into two queries using "CONDITION1" and "CONDITION2" respectively. They return with 2 set of records. The intersection of these two sets gives the result of the primitive query.

For example, the query demonstrated by example 3 can be divided into two queries as indicated by example 1 and example 2 respectively.

Fact 2: Assume that a query condition has the form of "CONDITION1 OR CONDITION2" . Similarly, the query can be separated into two queries, and the union of the set returned by them gives the result of the primitive query.

Fact 3: Assume that a query condition has the form of "NOT CONDITION". The result of the primitive query is the complementary set of the resulting set of records of the query with query condition "CONDITION".

With these three facts, Theorem 1 is obvious. Here, we only show how to split a complex query into simple query without considering the problem of query optimization. Then, how many potential target records in a simple query? It depends on both the content of the database we are manipulating and the query itself. In the next two subsections, we will consider how the effect of the content of the database and how a simple query can be converted into a sequence of calls to the factorized quantum search algorithm. But here, we return to summarize the function of the search engine. It can be described as follows:

a. Accept query request.

b. Call the analysis module to analyze the query condition.

c. Split the primitive query into simply queries.

d. Initiate a sequence of calls to the implementation module.

e. Collect the results returned by the calls and do further operations.

f. Return the final result.

In short, the quantum search engine will convert every complex query into a sequence of simple queries each with one logical expression, and finally into a sequence of calls to the factorized quantum search algorithm.

\section{B. Digitalization}

Digitization means writing all the property values as strings of letters belonging to a finite alphabet [1]. This requires that all the property values, among which the algorithm is going to searching for desired items, must be distinct from each other. Digitization on the key values is easy, because the key values are distinct. Consider the case that if the content of TABLE 1 is the content of the database we are going to query for target records. We can use a simple coding scheme for this case by replacing $960112 \sim 960115$ by $0 \sim 3$, and digitize them as

$$
\begin{aligned}
& 0 \longrightarrow|00\rangle \\
& 1 \longrightarrow|01\rangle \\
& 2 \longrightarrow|10\rangle \\
& 3 \longrightarrow|11\rangle .
\end{aligned}
$$

Digitization also requires that a database file must have $N=4^{n}$ items. If its original file does not meet this requirement, we have to pad up the database file. And we need a tag to identify whether or not a database file has been padded up. If implementation of factorized quantum search algorithm gives a record with tag indicating that it is a padding item, then the result would be abandoned. But for simplicity, we assume the requirement is satisfied (In fact, we can avoid this problem by allocating a database file with size $L * 4^{n}$, where $\mathrm{L}$ is the size of an item).

The values of common property are generally not distinct from each other in an actual database. A solution to this problem is that using one or more auxiliary files to record the values of common property if we permit a search on the property. If a common property has two or more auxiliary files (If the property values are distinct from each other, then it doesn't need any auxiliary file), we say that the database enable queries on the property. In other words, a search on the values of a common property without the help of auxiliary file will possibly fail. The auxiliary file has such a structure as

\section{\begin{tabular}{|l|l|}
\hline Property values & Addresses in main file \\
\hline
\end{tabular}}

Table. 5: Structure of an auxiliary file.

A table in a database corresponds to a main file and two or more auxiliary files. If the database detects that a manipulation on it will result in non-uniqueness of one or more property values, it will record the property value in an auxiliary file. It needs one or more auxiliary files for a property. The number of the auxiliary files corresponding to a property rests with how many property values are the same.

For example, to record the property values of "Age", it might need such two auxiliary files as 


\begin{tabular}{|c|c|}
\hline Property values & Addresses in main file \\
\hline 18 & 1 \\
\hline 20 & 2 \\
\hline 19 & 3 \\
\hline
\end{tabular}

Table. 6, A

\begin{tabular}{|c|c|}
\hline Property values & Addresses in main file \\
\hline 20 & 4 \\
\hline
\end{tabular}

Table. 6, B

Table. 6: Auxiliary files for table 1.

Here, we suppose the addresses of the four records in table 1 are $1,2,3$, and 4 . Note that all the property values in an auxiliary file should be distinct from each other. So we can treat the property values in a auxiliary file in the way as the key values when digitalizing.

\section{Initiation of calls to implementation module}

In this subsection, we focus on a simple query with only a logical expression as its query condition. We have said that a logical expression is an expression that has two operands connected with a logical operator from the set $\{>, \geqslant,<, \leqslant,=, \neq\}[$ [8]. A logical expression in a simply query has such a form as "x o a", where $\mathrm{x}$ is a property variable, $\mathrm{o}$ is a logical operator, and a is a given property value. Assume that the definition domain of $\mathrm{x}$ is $\left[x_{1}, x_{2}\right]$. The algorithm of initiating calls to implementation module can be described as the following subroutines each corresponding to a type of logical expression.

$\mathrm{f}(\mathrm{x},=, \mathrm{a})$ :

a. If $x$ is a key property, then call the implementation module with the query condition on the main file.

b. Else if the search on the property $\mathrm{x}$ is not allowable, i.e., its property values are not distinct from each other and no auxiliary file are available, then return with failure.

c. For Every auxiliary file, call the implementation module with the query condition.

d. Return with the union set of the results of all calls. $\mathrm{f}(\mathrm{x}, \neq, \mathbf{a})$ :

a. For every y in $\left[x_{1}, x_{2}\right]$ and unequal to a, call $\mathrm{f}(\mathrm{x},=, \mathrm{y})$.

b. Return with the union set of the results of all calls. $\mathrm{f}(\mathrm{x},<, \mathrm{a})$ :

a. For Every y in $\left[x_{1}, a\right)$, call $\mathrm{f}(\mathrm{x},=, \mathrm{y})$.

b. Return with the union set of the result of all calls.

The subroutines for the rest types of logical expression, i.e., $\mathrm{f}(\mathrm{x}, \leqslant, \mathrm{a}), \mathrm{f}(\mathrm{x},>, \mathrm{a})$, and $\mathrm{f}(\mathrm{x}, \geqslant, \mathrm{a})$, are similar to $\mathrm{f}(\mathrm{x},<, \mathrm{a})$.

\section{The implementation module}

We have known that the implementation module carrys out the factorized quantum search algorithm. So, we turn to see how the factorized quantum search algorithm work on an actual quantum computer. Assume that our database exists in a qRAM, the content of the database is table 1, and we want to find the record that "ID=960112". The four property values of "ID" are encoded as

$$
\begin{aligned}
& 960112 \longrightarrow|00\rangle \\
& 960113 \longrightarrow|01\rangle \\
& 960114 \longrightarrow|10\rangle \\
& 960115 \longrightarrow|11\rangle .
\end{aligned}
$$

First, we need to prepare the content of the database in a uniform state. we can do this job by setting the address register $\mathrm{A}$ in the state $\frac{1}{2}(|a\rangle+|b\rangle+|c\rangle+|d\rangle)$, where a, b, c, d, are the corresponding addresses of the four records. And the qRAM will return a superposition of data in a data register $\mathrm{D}$, correlated with the address register

$$
\frac{1}{2}(|00\rangle \otimes|a\rangle+|01\rangle \otimes|b\rangle+|10\rangle \otimes|c\rangle+|11\rangle \otimes|d\rangle)
$$

It is easy to see that it takes only one oracle query for the factorized quantum search algorithm to find the desired item, and the resulting state of the register would be $|00\rangle \otimes|a\rangle$. Measurement on the register gives the desired item with certainty.

\section{E. query complexity and space consumption}

If a property has $\mathrm{t}$ possible values, and $j_{i}$ denotes the number of items with the property value $p_{i}$, where $\mathrm{i}$ ranges from 1 to $t$, then the number of the auxiliary files is $M=\max \left(j_{1}, j_{2}, \ldots, j_{t}\right)$. Let the size of the $\mathrm{M}$ auxiliary files be $N_{1}, N_{2}, \ldots, N_{M}$, then the total memory space for all the M auxiliary files is $N_{1}+N_{2}+\ldots+N_{M}$, which is $4 N$ in the worst case (Note that we need to pad every auxiliary file such that $N_{i}$ is the power of 4 ).

Theorem 2. The query complexity of Our algorithm for the quantum search engine responding to one complex query request is at most $O\left(P * Q * M * \log _{4} N\right)$, where $P$ is the number of the potential simple query requests in the complex query request, $Q$ is the maximum number of calls to the factorized quantum search algorithm, $M$ is the number of the auxiliary files for the property on which our algorithm are searching for desired items.

Proof: Because a complex query is converted into $P * Q$ calls to the factorized quantum search algorithm on every auxiliary files, the total number of calls to the factorized quantum search algorithm is $P * Q * M$. And the query 
complexity of the factorized quantum search algorithm is $O\left(\log _{4} N\right)$ [1]. So, the total query complexity corresponding to a complex query is at most $O\left(P * Q * M * \log _{4} N\right)$.

If the values of the property, on which our algorithm are implementing, are distinct from each other, then the query complexity are $O\left(P * Q * \log _{4} N\right)$. However, the query complexity of a typical search engine using the best classical search algorithm mentioned above is $O(P *$ $\left.Q * \log _{2} N\right)$ for a complex query.

\section{EXAMPLES}

We take the example 3 in section II as an example to show how our quantum search engine works. After the engine accept the query request, it will first call the analysis module to analyze the query condition, and split the primitive query into simply queries demonstrated by example 1 and 2. Let us see how the engine deals with the example 1 and 2. Assume that "ID" is the key property and the database enable queries on the property "Age".

\section{Search process of example 1:}

a. The engine calls the subroutine $\mathrm{f}($ Age,$=, 20)$.

b. Because "Age" is not the key property, then for every auxiliary files(The auxiliary files can be seen in table 6.), call the implementation module with the query condition.

c. For the first auxiliary file, the implementation module returns with

\begin{tabular}{|c|c|c|}
\hline ID & Name & Age \\
\hline 960114 & Yang & 20 \\
\hline
\end{tabular}

For the second auxiliary file, the implementation module returns with

\begin{tabular}{|c|c|c|}
\hline ID & Name & Age \\
\hline 960115 & Yingyu & 20 \\
\hline
\end{tabular}

d. The result of example 1 , which can seen in table 2 , is the union of these two results.

Search process of example 2:

a. The engine calls the subroutine $\mathrm{f}(\mathrm{ID},<, 960115)$.

b. The subroutine then call $\mathrm{f}(\mathrm{ID},=, 960112)$, $\mathrm{f}(\mathrm{ID},=960113)$, and $\mathrm{f}(\mathrm{ID},=, 960114)$. Note that ID is a key property, then $\mathrm{f}(\mathrm{ID},=, 960112)$ and $\mathrm{f}(\mathrm{ID},=, 960113)$ call the implementation module with the corresponding query condition respectively on the main file.

c. $\mathrm{f}(\mathrm{ID},=, 960112)$ returns with

\begin{tabular}{|c|c|c|}
\hline ID & Name & Age \\
\hline 960112 & Lin & 18 \\
\hline
\end{tabular}

$\mathrm{f}(\mathrm{A},=, 960113)$ returns with

\begin{tabular}{|c|c|c|}
\hline ID & Name & Age \\
\hline 960113 & Lin & 19 \\
\hline
\end{tabular}

$\mathrm{f}(\mathrm{A},=, 960114)$ returns with

\begin{tabular}{|c|c|c|}
\hline ID & Name & Age \\
\hline 960114 & Lin & 20 \\
\hline
\end{tabular}

The union of the results of $\mathrm{f}(\mathrm{ID},=, 960112)$, $\mathrm{f}(\mathrm{A},=, 960113)$, and $\mathrm{f}(\mathrm{A},=, 960114)$ gives the results of example 2, which can be seen in table 3 . And the intersection of the results of example 1 and 2 is the result of example 3 , which can be seen in table 4 .

\section{CONCLUSION}

We have considered the problem of finding one or more desired items out of an unsorted database. The factorized quantum search algorithm presented by Patel can locate one desired item in an unsorted database using $\mathrm{O}(\log 4 \mathrm{~N})$ queries to factorized oracles. But the algorithm can only solve the problem finding the desired item out of an unsorted database with only one target item. We extend the algorithm to solve the problem with more than one target items in an unsorted database. The goal is achieved by introducing auxiliary file, and converting a complex query request into a sequence of simple queries, and finally into a sequence of calls to the factorized quantum search algorithm.

The query complexity of our algorithm is $O(P * Q *$ $\left.M * \log _{4} N\right)$, where $\mathrm{P}$ is the number of the potential simple query requests in the complex query request, $\mathrm{Q}$ is the maximum number of calls to the factorized quantum search algorithm of the simple queries, $M$ is the number of the auxiliary files for the property on which our algorithm are searching for desired items. We know that the query complexity of the best classical algorithm to select $\mathrm{P}^{*} \mathrm{Q}$ items from a sorted database is $P * Q * \log _{2} N$. When compared with the classical algorithm on either sorted or unsorted database, if $M$ is small, the quantum search engine on unsorted database presented in the paper is more efficient.

The most important thing implied in this paper is that to manage an unsorted database on a quantum computer is possible and efficient. Though a query algorithm on a sorted database can be more efficient than a query algorithm on an unsorted database, the sorting price might be very high. So the quantum search algorithm presented in this paper offers another way to database management on an actual quantum computer. 
(2000).

[3] E. Farhi, J. Goldstone, S. Gutmann, and M. Sipser, arXiv:quant-ph/0009032 (1999).

[4] A. M. Childs, A. J. Landahl, and P. A. Parrilo, Phys. Rev. A 75, 032335 (2007).

[5] M. A. Nielson and I. L. Chuang, Quantum Computation and Quantum Information (Cambridge University Press, Cambridge, 2000).

[6] V. Giovannetti, S. Lloyd, and L. maccone, Phys. Rev.
Lett 100, 160501 (2008).

[7] R. R. Elmasri and S. B. Navathe, Fundamentals of Database System (Addison Wesley, Boston, 2006).

[8] A. Younes, arXiv e-Print quant-ph/0705.4303 (2007).

[9] L. K. Grover, in Proceedings of 28th Annual ACM Symposium on Theory of Computing (1996), pp. 212-219.

[10] L. K. Grover, Phys. Rev. Lett. 79, 325 (1997). 